\title{
Optimum Groove Location of Hydrodynamic Journal Bearing Using Genetic Algorithm
}

\author{
Lintu Roy and S. K. Kakoty \\ Indian Institute of Technology Guwahati, Guwahati 781309, India \\ Correspondence should be addressed to S. K. Kakoty; sashin@iitg.ernet.in
}

Received 15 February 2013; Revised 13 May 2013; Accepted 31 May 2013

Academic Editor: Michel Fillon

Copyright ( 2013 L. Roy and S. K. Kakoty. This is an open access article distributed under the Creative Commons Attribution License, which permits unrestricted use, distribution, and reproduction in any medium, provided the original work is properly cited.

This paper presents the various arrangements of grooving location of two-groove oil journal bearing for optimum performance. An attempt has been made to find out the effect of different configurations of two groove oil journal bearing by changing groove locations. Various groove angles that have been considered are $10^{\circ}, 20,^{\circ}$ and $30^{\circ}$. The Reynolds equation is solved numerically in a finite difference grid satisfying the appropriate boundary conditions. Determination of optimum performance is based on maximization of nondimensional load, flow coefficient, and mass parameter and minimization of friction variable using genetic algorithm. The results using genetic algorithm are compared with sequential quadratic programming (SQP). The two grooved bearings in general have grooves placed at diametrically opposite directions. However, the optimum groove locations, arrived at in the present work, are not diametrically opposite.

\section{Introduction}

Journal bearings are used extensively in rotating machines because of their low wear and good damping characteristics. Fluid-film journal bearings are available to support a rotating shaft in a turbo machinery system. A full circular journal bearing has a much simple configuration but exhibits instability at higher rotational speeds. It is relatively less expensive compared to the multilobe bearings. It is well known that whirl instability occurs at high speed in oil journal bearing. Present day bearings, at over increasing speeds and loads, confront the engineer with many new problems. Excessive power losses reduce the efficiency of the engine, and high bearing temperature poses a danger to material of the bearing as well as the lubricant. Instability arising mainly in the form of oil whip may ruin not only the bearing but the machine itself. New bearing designs are sought to meet the new requirements. A journal bearing fed by two axial grooves has a wide practical application due to its good load carrying capacity and ability to operate when reversal of shaft rotation occurs [1]. These bearing usually have the grooves positioned orthogonal to the predominant load direction. Among the previous works on two axial groove oil journal bearings; Klit and Lund [2] used finite element method to find dynamic coefficients of plain circular bearing with two $20^{\circ}$ axial grooves. Gethin and Deihi [3] studied the effect of loading direction on the performance of a twin-axial groove cylindrical bore bearing. It has been anticipated that, if the bearing is loaded into the groove, its load carrying ability will be diminished, but the effect on hydrodynamic lubricant flow and power loss is not so obvious. If the positions of the grooves are arranged for carrying a relatively higher load, then the likelihood of bearing instability reduces, since the journal will run more eccentrically. Again hydrodynamic leakage and friction are affected by the direction of loading. So a question arises: where the position of the groove should lie so as to give the optimum load capacity, flow, friction, and critical speed. A new technique for optimizing hybrid journal bearings was presented by Rowe and Koshal [4]. The method involved the comparison of the bearings to be optimized with a reference bearing on the basis of load/total power, load/pumping power, and load/flow. Lin and Noah [5] used genetic algorithm to optimize the performance of a hydrodynamic journal bearing. Hashimoto and Matsumoto [6] described the optimum design methodology for improving operating 
characteristics of hydrodynamic journal bearings. The hybrid optimization technique combining the direct search method and the successive quadratic programming has been applied to find the optimum design of elliptical journal bearings. Boedo and Eshkabilov [7] described the implementation of a genetic algorithm suitable for the optimal shape design of finite-width, isoviscous, fluid film journal bearings under steady load and steady journal rotation. Hirani [8] formulated a problem to minimize temperature rise, power loss, and oil flow. An evolution-based optimization methodology for cylindrical journal bearings had been applied for journal bearings.

David et al. [9] in their paper presented the basic concepts of traditional genetic algorithm, its advantages with variety of applications. The paper also pointed out advanced features and future directions. McCall [10] presented genetic algorithms (GAs), a heuristic search and optimization technique inspired by natural evolution. GAs have been successfully applied to a wide range of real-world problems of significant complexity. When there are hundreds of publications on application of GAs, only couple of representative publications are cited here.

It has been observed that GAs have been successfully applied for optimizing bearing performance. However, the performance of two-groove journal bearing has not been optimized pertaining to location of groove positions with multiple objectives. In view of this, an attempt has been made in this paper to obtain an optimum configuration of the two grooves positions around the circumference of the hydrodynamic journal bearing for maximum oil flow, minimum friction loss, maximum load bearing capacity, and maximum critical speed vis-à-vis mass parameter, a function of speed.

1.1. Oil Flow. The oil flow rate depends on several factors, such as the viscosity of the lubricant, the geometry (length, diameter, and radial clearance) of the bearing, operating eccentricity, the inlet oil pressure, the arrangement of feeding sources, and groove location of the bearing. The pressure developed in the film due to journal motion also contributes to the flow. An adequate oil flow takes away frictional heat and does not allow rapid rise in temperature.

1.2. Friction Loss. The calculation of friction loss within a bearing oil film is an integral part of the design of the bearing. The friction loss appears as heat, raises the temperature of the lubricant and lowers its viscosity, which is a key parameter of the bearing analysis. Therefore, the accurate prediction of friction loss is desired. The friction force is calculated by integrating shear stress over the journal surface. It is desired to keep the friction loss at minimum.

1.3. Load Carrying Capacity. The load carrying capacity of the bearing within a bearing is developed due to pressure developed in the film. For a more accurate analysis, careful consideration of film extent needs to be included. This is expected to influence hydrodynamic leakage significantly and load carrying ability under some circumstances. If the feeding groove (in which pressure is zero) falls in the load carrying film, this part of the bearing makes no contribution to the load-carrying ability. Thus the location of the groove plays a role in determining the load carrying ability of the bearing.

1.4. Critical Speed of Instability. Plain circular bearing is mostly replaced by some other bearings, as plain bearing does not suit the stability requirements of high-speed machines and precision machine tools. Grooved circular bearings and multilobe bearings with two lobes, three lobes, and four lobes are commonly used. The critical mass parameter (a measure of stability) is a function of speed. The higher the critical speed is, the higher the stability limit is. The larger the eccentricity ratio is, the more stable the shaft is. If the eccentricity ratio is larger than 0.8 , in particular, the shaft is always stable. In engineering analysis it is essential to know the critical speed at which oil whirl occurs and avoid it during operation. It has been found that severe whirl occurs when the shaft speed is approximately twice the bearing critical frequency.

1.5. Selection Procedure. To facilitate the optimum bearing design in the present paper, the nondimensional values of flow coefficient, load, and mass parameters along with friction variables for different configurations in groups are estimated. The optimum performance is determined on the basis of maximization of flow, load, mass parameter, and minimization of friction variable.

\section{Theory}

The Reynolds equation in two dimensions for an incompressible fluid is the governing equation. It can be written in a dimensionless form as

$$
\frac{\partial}{\partial \theta}\left(\bar{h}^{3} \frac{\partial \bar{p}}{\partial \theta}\right)+\left(\frac{D}{L}\right)^{2}\left(\bar{h}^{3} \frac{\partial^{2} \bar{p}}{\partial \bar{z}^{2}}\right)=\frac{\partial \bar{h}}{\partial \theta}+2 \lambda \frac{\partial \bar{h}}{\partial \tau}
$$

where,

$$
\begin{gathered}
\theta=\frac{x}{R}, \quad \bar{z}=\frac{z}{L / 2}, \quad \bar{h}=\frac{h}{C}, \quad \bar{p}=\frac{p C^{2}}{6 \eta U R}, \\
\tau=\omega_{p} t, \quad \lambda=\frac{\omega_{p}}{\omega} .
\end{gathered}
$$

The pressure and film thickness can be expressed for small amplitude of vibration as

$$
\begin{gathered}
\bar{p}=\bar{p}_{0}+\varepsilon_{1} e^{i \tau} \bar{p}_{1}+\varepsilon_{0} \phi_{1} e^{i \tau} \bar{p}_{2}, \\
\bar{h}=\bar{h}_{0}+\varepsilon_{1} e^{i \tau} \cos \theta+\varepsilon_{0} \phi_{1} e^{i \tau} \sin \theta .
\end{gathered}
$$

Substitution of (3) into (1) and retaining the first order terms and by equating the coefficients of $\varepsilon_{0}, \varepsilon_{1} e^{i \tau}$, and $\varepsilon_{0} \phi_{1} e^{i \tau}$, three 
differential equations in $\bar{p}_{0}, \bar{p}_{1}$, and $\bar{p}_{2}$ are obtained as shown in the following:

$$
\begin{gathered}
\frac{\partial}{\partial \theta}\left(\bar{h}_{0}^{3} \frac{\partial \bar{p}_{0}}{\partial \theta}\right)+\left(\frac{D}{L}\right)^{2} \frac{\partial}{\partial \bar{z}}\left(\bar{h}_{0}^{3} \frac{\partial \bar{p}_{0}}{\partial \bar{z}}\right)=\frac{\partial \bar{h}_{0}}{\partial \theta}, \\
\frac{\partial}{\partial \theta}\left(\bar{h}_{0}^{3} \frac{\partial \bar{p}_{1}}{\partial \theta}\right)+\left(\frac{D}{L}\right)^{2} \frac{\partial}{\partial \bar{z}}\left(\bar{h}_{0}^{3} \frac{\partial \bar{p}_{1}}{\partial \bar{z}}\right) \\
+3 \frac{\partial}{\partial \theta}\left(\bar{h}_{0}^{2} \frac{\partial \bar{p}_{0}}{\partial \theta} \cos \theta\right)+\left(\frac{D}{L}\right)^{2} \frac{\partial}{\partial \bar{z}}\left(\bar{h}_{0}^{2} \frac{\partial \bar{p}_{0}}{\partial \bar{z}} \cos \theta\right) \\
=-\sin \theta+i 2 \lambda \cos \theta, \\
\frac{\partial}{\partial \theta}\left(\bar{h}_{0}^{3} \frac{\partial \bar{p}_{2}}{\partial \theta}\right)+\left(\frac{D}{L}\right)^{2} \frac{\partial}{\partial \bar{z}}\left(\bar{h}_{0}^{3} \frac{\partial \bar{p}_{2}}{\partial \bar{z}}\right) \\
+3 \frac{\partial}{\partial \theta}\left(\bar{h}_{0}^{2} \frac{\partial \bar{p}_{0}}{\partial \theta} \sin \theta\right)+\left(\frac{D}{L}\right)^{2} \frac{\partial}{\partial \bar{z}}\left(\bar{h}_{0}^{2} \frac{\partial \bar{p}_{0}}{\partial \bar{z}} \sin \theta\right) \\
=-\cos \theta+i 2 \lambda \sin \theta .
\end{gathered}
$$

Boundary conditions used for the steady state pressure and dynamic pressure distribution are as follows:

$$
\begin{gathered}
\frac{\partial \bar{p}_{i}}{\partial \theta}=0, \quad \bar{p}_{i}=0 \text { at } \theta=\theta_{r}, \\
\bar{p}_{i}(\theta, \bar{z})=0, \quad \text { when } \theta_{s} \leq \theta \leq \theta_{e},
\end{gathered}
$$

where, $\bar{p}_{i}=\bar{p}_{0}, \bar{p}_{1}, \bar{p}_{2}$ and $\theta_{s}$ : starting angle of the groove with respect to the vertical axis, $\theta_{e}$ : angle at which the groove ends with respect to the vertical axis, and $\theta_{r}$ : angle at which the film cavitates with respect to the vertical axis.

The nondimensional steady state load components as well as the nondimensional steady state load are given by

$$
\begin{gathered}
\bar{W}_{X_{0}}=\int_{\theta_{1}}^{\theta_{2}} \int_{0}^{1} \bar{p}_{0} \cos \theta d \theta d \bar{z}, \\
\bar{W}_{Z_{0}}=\int_{\theta_{1}}^{\theta_{2}} \int_{0}^{1} \bar{p}_{0} \sin \theta d \theta d \bar{z}, \\
\bar{W}_{0}=\sqrt{\bar{W}_{X_{0}}^{2}+\bar{W}_{Z_{0}}^{2}} .
\end{gathered}
$$

Equation (4) is solved for the steady state pressure distribution $\left(\bar{p}_{0}\right)$, discretizing in a finite difference grid of size $88 \times 14$ and using Gauss-Seidel method with successive overrelaxation (SOR) technique satisfying the boundary conditions. The convergence criterion adopted for pressure calculation is $\left|1-\sum \bar{p}_{\text {old }} / \sum \bar{p}_{\text {new }}\right| \leq 10^{-5}$. Chosen bearing eccentricity and arbitrary attitude angle picked at random result in magnitude of forces generated due to pressure wedge in the bearing. The attitude angle is changed till the horizontal force component $\left(\bar{W}_{Z_{0}}\right)$ in the pressure wedge becomes zero. This eventually locates the attitude angle. For this equilibrium position the vertical force $\left(\bar{W}_{X_{0}}\right)$ gives the load capacity, $\bar{W}_{0}$. The Sommerfeld number is given by $S=1 / \pi \bar{W}_{0}$.
The flow coefficient in the dimensionless form can be written as

$$
\bar{q}_{Z}=\frac{1}{2}\left(\frac{D}{L}\right)^{2} \int_{0}^{2 \pi} \bar{h}_{0}^{3} \frac{\partial \bar{p}_{0}}{\partial \bar{z}} d \theta .
$$

The friction variable is given by

$$
\bar{\mu}=\mu\left(\frac{R}{C}\right)=\frac{\int_{0}^{2 \pi}\left(3 \bar{h}\left(\partial \bar{p}_{0} / \partial \theta\right)+1 / \bar{h}\right) d \theta}{6 \bar{W}} .
$$

Equations (5) and (6) for $\bar{p}_{1}$ and $\bar{p}_{2}$ are solved satisfying the boundary conditions and known values of $\bar{p}_{0}$ using the same procedure used for calculating steady state pressure. Dynamic loads due to $\bar{p}_{1}$ and $\bar{p}_{2}$ are given by

$$
\begin{aligned}
& \bar{W}_{X 1}=\int_{\theta_{1}}^{\theta_{2}} \int_{0}^{1} \bar{p}_{1} \cos \theta d \theta d \bar{z}, \\
& \bar{W}_{Z 1}=\int_{\theta_{s}}^{\theta_{e}} \int_{0}^{1} \bar{p}_{1} \sin \theta d \theta d \bar{z}, \\
& \bar{W}_{X 2}=\int_{\theta_{1}}^{\theta_{2}} \int_{0}^{1} \bar{p}_{2} \cos \theta d \theta d \bar{z}, \\
& \bar{W}_{Z 2}=\int_{\theta_{s}}^{\theta_{e}} \int_{0}^{1} \bar{p}_{2} \sin \theta d \theta d \bar{z} .
\end{aligned}
$$

It is found that the fluid film, which supports the bearing, is equivalent to a spring mass damping system. Since the journal executes small harmonic oscillations about its steady state position, the dynamic load carrying capacity can be expressed as a spring and a viscous damping force. The stiffness and damping coefficients are given by

$$
\begin{array}{ll}
\bar{K}_{X X}=-\operatorname{Re}\left(\bar{W}_{X 1}\right) ; & \bar{K}_{Z X}=-\operatorname{Re}\left(W_{Z t 1}\right) ; \\
\bar{K}_{X Z}=-\operatorname{Re}\left(\bar{W}_{X t 2}\right) ; & \bar{K}_{Z Z}=-\operatorname{Re}\left(\bar{W}_{Z t 2}\right), \\
\bar{C}_{X X}=-\operatorname{Im}\left(\bar{W}_{X t 1}\right) ; & \bar{C}_{Z X}=-\operatorname{Im}\left(\bar{W}_{Z t 1}\right) ; \\
\bar{C}_{X Z}=-\operatorname{Im}\left(\bar{W}_{X t 2}\right) ; & \bar{C}_{Z Z}=-\operatorname{Im}\left(\bar{W}_{Z t 2}\right) .
\end{array}
$$

2.1. Mass Parameter and Whirl Ratio. The nondimensional linearised equations of journal motion can be written as [11]

$$
\begin{aligned}
& \bar{M} \Delta \bar{X}_{0}+\bar{K}_{X X} \Delta \bar{X}+\bar{K}_{X Z} \Delta \bar{Z}+\bar{C}_{X X} \Delta \bar{X}+\bar{C}_{X Z} \Delta \overline{\dot{Z}}=0, \\
& \bar{M} \Delta \bar{Z}_{0}+\bar{K}_{Z X} \Delta \bar{X}+\bar{K}_{Z Z} \Delta \bar{Z}+\bar{C}_{Z X} \Delta \overline{\dot{X}}+\bar{C}_{Z Z} \Delta \overline{\dot{Z}}=0,
\end{aligned}
$$

where $\bar{M}=m C \omega^{2} / W$, a nondimensional mass parameter.

Now, $\left(\bar{x}_{0}, \bar{z}_{0}\right)$ is the steady state equilibrium position (nondimensional) of the journal. $(\Delta \bar{X}, \Delta \bar{Z})$ is the perturbed amount from this position at a nondimensional time " $\tau$ ". The instantaneous positions are given by

$$
\begin{array}{rlrl}
\bar{x}=\bar{x}_{0} e^{i \lambda \tau}, & \bar{z}=\bar{z}_{0} e^{i \lambda \tau}, \\
\dot{\bar{x}}=i \lambda \bar{x}_{0} e^{i \lambda \tau}, & & \dot{\bar{z}}=i \lambda \bar{z}_{0} e^{i \lambda \tau}, \\
\ddot{\bar{x}}=-\lambda^{2} \bar{x}_{0} e^{i \lambda \tau}, & & \ddot{\bar{z}}=-\lambda^{2} \bar{z}_{0} e^{i \lambda \tau} .
\end{array}
$$


Substituting the above nondimensional terms in the equations of motion (see (15)), a characteristic equation is formed to find a non-trivial solution. Solving the characteristic equation, the following expressions for the mass parameter, $\bar{M}$, and the whirl ratio, $\lambda$, are arrived at

$$
\lambda^{2} \bar{M}=\frac{\bar{K}_{X X} \bar{C}_{Z Z}+\bar{K}_{Z Z} \bar{C}_{X X}-\left(\bar{K}_{X Z} \bar{C}_{Z X}+\bar{K}_{Z X} \bar{C}_{X Z}\right)}{\bar{C}_{X X}+\bar{C}_{Z Z}}=k_{0} .
$$

So,

$$
\lambda^{2}=\frac{\left(\bar{K}_{X X}-k_{0}\right)\left(\bar{K}_{Z Z}-k_{0}\right)-\bar{K}_{X Z} \bar{K}_{Z X}}{\bar{C}_{X X} \bar{C}_{Z Z}-\bar{C}_{X Z} \bar{C}_{Z X}}, \quad \bar{M}=\frac{k_{0}}{\lambda^{2}} .
$$

\section{Optimization Techniques}

It has been found that the location of the groove has an influence on flow $\left(\bar{q}_{Z}\right)$, frictional variable $(\bar{\mu})$, load carrying capacity $(\bar{W})$, and mass parameter $(\bar{M})$. Genetic algorithm (GA) is the most popular stochastic method used to find the optimum solution for all kinds of problems. The most striking difference between GAs and many traditional optimization methods is that GAs work with a population of points instead of a single point. On the other hand, since GAs require only function values at various discrete points, a discrete or discontinuous function can be handled with no extra burden. This allows GAs to be applied to a wide variety of problems. Another advantage with a population-based search algorithm is that multiple optimal solutions can be captured in the population easily, thereby reducing the effort to use the same algorithm many times. Genetic algorithms perform a multiple directional search by maintaining a population of potential solutions. The population-to-population approach attempts to make the search escape from local optima [6]. GAs are very helpful when the developer does not have precise domain expertise because GAs possess the ability to explore and learn from their domain.

3.1. Multiobjective Problem Formulation. The problem is framed with four objectives. The variables used in the problem are in case-I starting angle of first groove $\left(\theta_{1}\right)$, starting angle of second groove $\left(\theta_{2}\right)$. The optimum configurations obtained for an eccentricity ratio range from 0.1 to 0.9 in this case. In case-II, the eccentricity ratio $(\varepsilon)$, starting angle of first groove $\left(\theta_{1}\right)$, and starting angle of second groove $\left(\theta_{2}\right)$ are variables and act as chromosome, the groove angles being $10^{\circ}$ in both cases. It has been found that for $10^{\circ}$ groove angle the pressure development as well as load carrying capacity is higher in comparison with $20^{\circ}$ and $30^{\circ}$. The objectives are minimization of friction variable $(\bar{\mu})$, Equation (12), maximization of load capacity $(\bar{W})$, Equation (10), flow coefficient $\left(\bar{q}_{Z}\right)$, Equation (11), maximization of mass parameter $(\bar{M})$, and Equation (17); objective function framing is same for both cases, and variable bounds are shown in Table 1 .
TABLE 1: Variable bounds for the bearing problem.

\begin{tabular}{llcc}
\hline Case & Variable & $\begin{array}{r}\text { Lower } \\
\text { bound }\end{array}$ & $\begin{array}{r}\text { Upper } \\
\text { bound }\end{array}$ \\
\hline I & Starting angle of first groove & $0^{\circ}$ & $180^{\circ}$ \\
& Starting angle of second groove & $170^{\circ}$ & $350^{\circ}$ \\
\hline \multirow{3}{*}{ II } & $\mathcal{E}$ & 0.1 & 0.9 \\
& Starting angle of first groove & $0^{\circ}$ & $180^{\circ}$ \\
& Starting angle of second groove & $170^{\circ}$ & $350^{\circ}$ \\
\hline
\end{tabular}

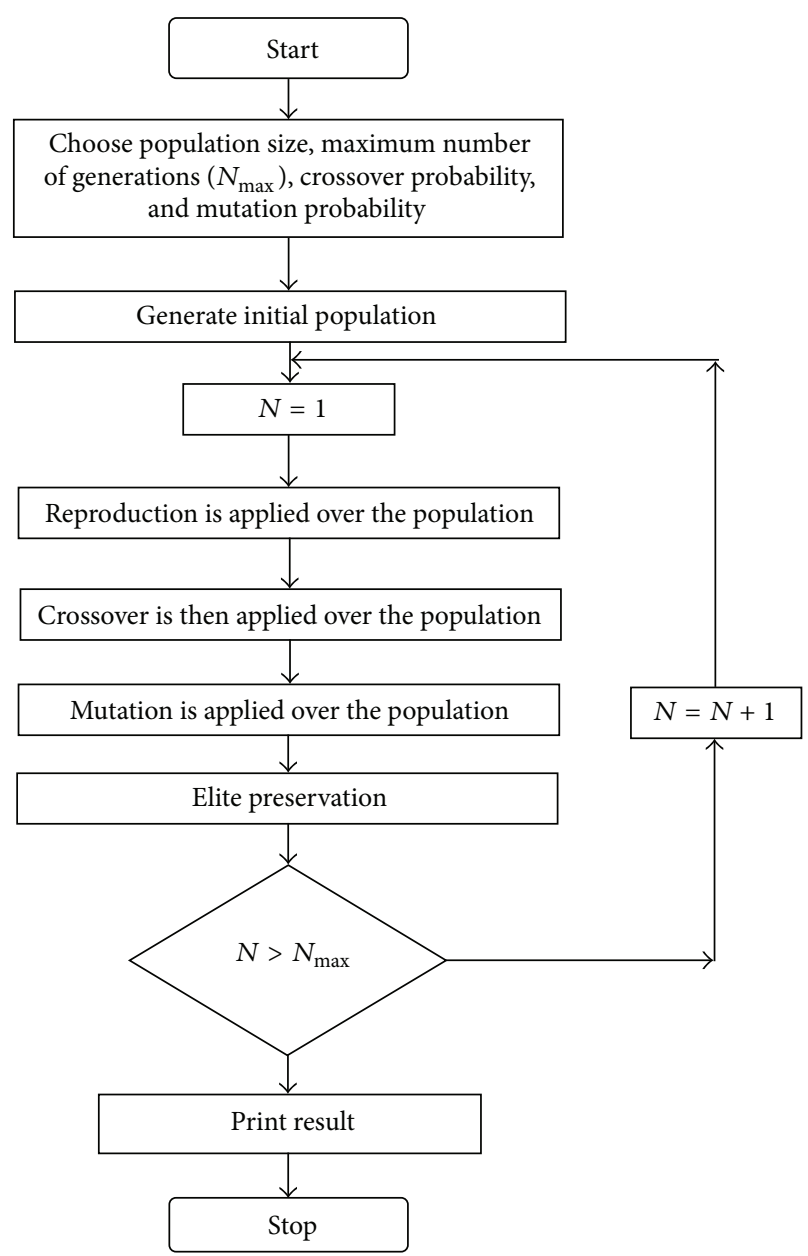

FIGURE 1: Flow chart for real-coded genetic Algorithm.

3.2. Real-Coded Genetic Algorithm Computational Procedure. In this problem three variables called genes will form a chromosome. A set of chromosome is called population. With uniform probability distribution all chromosomes in the population are initialized. The population of each generation will have feasible design variables (chromosome) in terms of their allowable ranges but may be infeasible otherwise. The main steps involved in the genetic algorithm are discussed below and shown in flow chart (Figure 1).

Real-oded GA comprises of mainly six steps as follows.

Step 1. There are mainly four user-defined parameters in the program, population size, maximum number of generation, 
cross over probability, and mutation probability. The best value of population size is 50 . It is found that the program is converging very fast with these values. Cross over probability and mutation probability are more sensitive parameters for this program.

Step 2. Second stage of program is to initialize the population size. So, 50 chromosomes are initialized using random probability for each variable span.

Step 3. The selection operator involves randomly choosing members of the population to enter a mating pool. The operator is carefully formulated to ensure that better members of the population (with higher fitness) have a greater probability of being selected for mating, but that worse members of the population still have a small probability of being selected. Having some probability of choosing worse members is important to ensure that the search process is global and does not simply converge to the nearest local optimum. Selection is one of the important aspects of the GA process, and there are several ways for the selection.

Step 4. Recombination is carried out through crossover and mutation operation in GA. The crossover operator is a method for sharing information between chromosomes. It ensures that the probability of reaching any point in the search space is never zero. The crossover operator is the main search operator in the GA. The search power of a crossover operator is defined as a measure of how flexible the operator is to create an arbitrary point in the search space. Crossover is useful in problems where building block exchange is necessary. It has been found that GAs may work well with large crossover probability and with a small mutation probability. A single point crossover preserves the structure of the parent string to the maximum. From a set of crossover operator, linear, blended crossover, and simulated binary crossover operators, it is found that, from trial run, the simulated binary crossover gives better convergence in limited time.

Step 5. From biological view, mutation is any change of DNA material that can be reproduced. From computer science view, mutation is a genetic operator that follows crossover operator. It usually acts on only one individual chosen based on a probability or fitness function. One or more genetic components of the individual are scanned. And this component is modified based on some user-definable probability or condition. Without mutation, offspring chromosomes would be limited to only the genes available within the initial population. Mutation should be able to introduce new genetic material as well as modify the existing one. With these new gene values, the genetic algorithm may be able to arrive at a better solution than was previously possible. Mutation operator prevents premature convergence to local optima by randomly sampling new points in the search space. There are many types of mutation, and these types depend on the representation itself. Random mutation finds a better suitability with the existing problem.

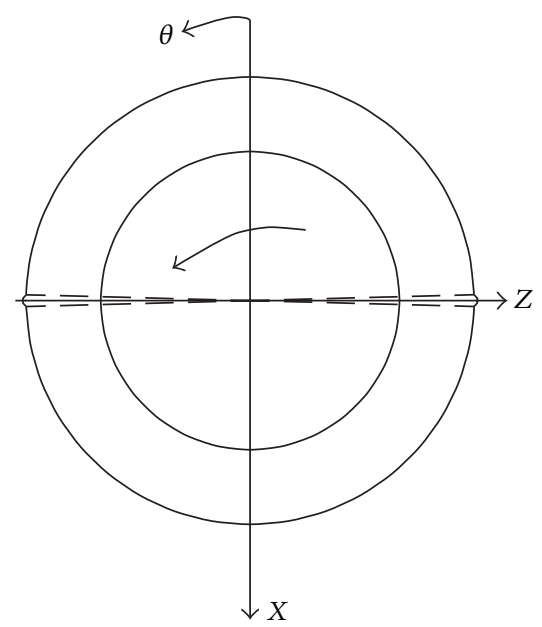

FIGURE 2: Hz-Hz configuration of two-groove oil journal bearing.

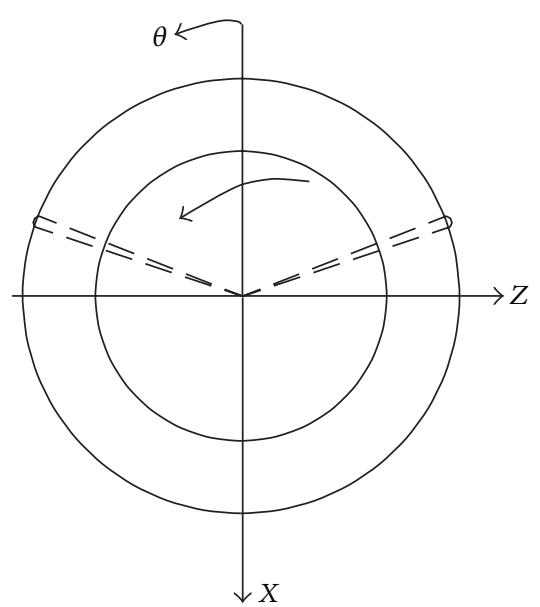

FIgURE 3: Up-Up configuration of two-groove oil journal bearing.

Step 6. Elite preservation forms a new population from the initial population and mutated one. This operator is responsible for convergence of the fitness by allowing better value to pass to the next generation.

\section{Results and Discussion}

The groove position located around the circumference is grouped as follows.

Group-I: Hz-Hz configuration: grooves are placed in a horizontal position $180^{\circ}$ apart, that is, diametrically opposite to each other (Figure 2).

Group-II: Up-Up configuration: both grooves are placed $\left(5^{\circ}\right.$ to $\left.80^{\circ}\right)$ above the horizontal position (Figure 3), and groove position is varied at $5^{\circ}$ interval. Up-Up-10 configuration means that both grooves are $10^{\circ}$ above the horizontal as shown in Figure 3.

Group-III: Up-Hz configuration: the left groove is $\left(5^{\circ}\right.$ to $80^{\circ}$ ) above the horizontal position, and the other groove is in horizontal position (Figure 3). Groove 


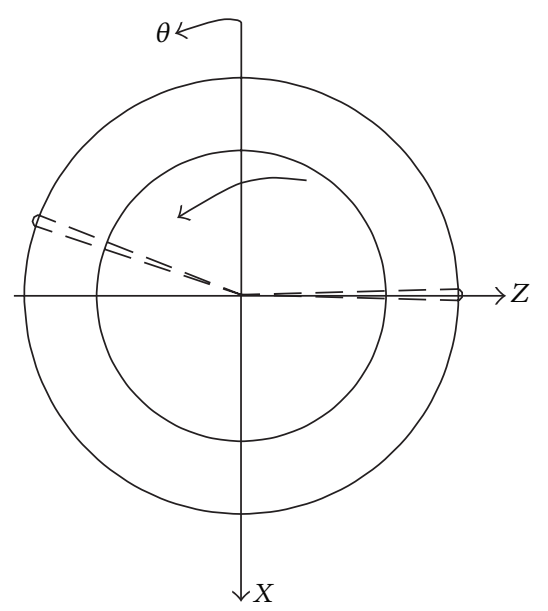

FIGURE 4: Up-Hz configuration of two-groove oil journal bearing.

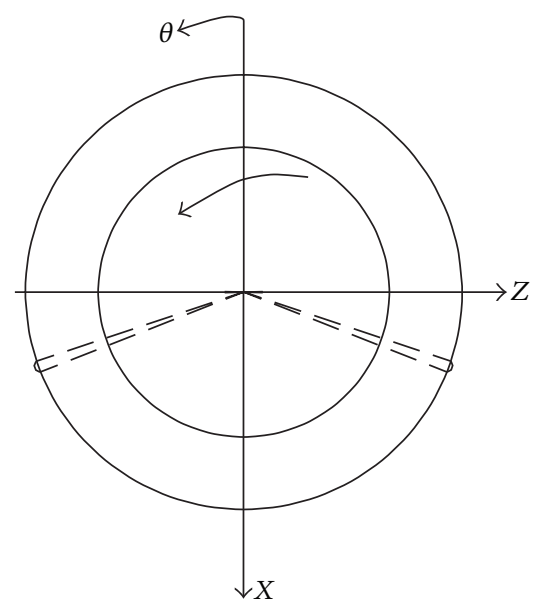

FIGURE 5: Dn-Dn configuration of two-groove oil journal bearing.

position is varied at $5^{\circ}$ interval (Figure 4). Up- $10 \mathrm{~Hz}$ configuration means that the left groove is $10^{\circ}$ above the horizontal and the right groove is horizontal as shown in Figure 4.

Group-IV: Dn-Dn configuration: both grooves are placed $\left(5^{\circ}\right.$ to $\left.80^{\circ}\right)$ below the horizontal position (Figure 5), and groove position is varied at $5^{\circ}$ interval. Dn-Dn-10 configuration means that both grooves are $10^{\circ}$ below the horizontal as shown in Figure 5.

Group-V: Hz-Up configuration: the left groove is in horizontal position, and the other groove is $\left(5^{\circ}\right.$ to $80^{\circ}$ ) above the horizontal position (Figure 6) groove position is varied at $5^{\circ}$ interval (Group-V). Hz-Up-10 configuration means that the left groove is horizontal and the right one is $10^{\circ}$ above the horizontal as shown in Figure 6.

Group-VI: Dn-Up configuration: one of the grooves is $\left(5^{\circ}\right.$ to $\left.80^{\circ}\right)$ below the horizontal position (the left one), and the other groove (the right one) is ( $5^{\circ}$ to $80^{\circ}$ ) above the horizontal position; groove position is

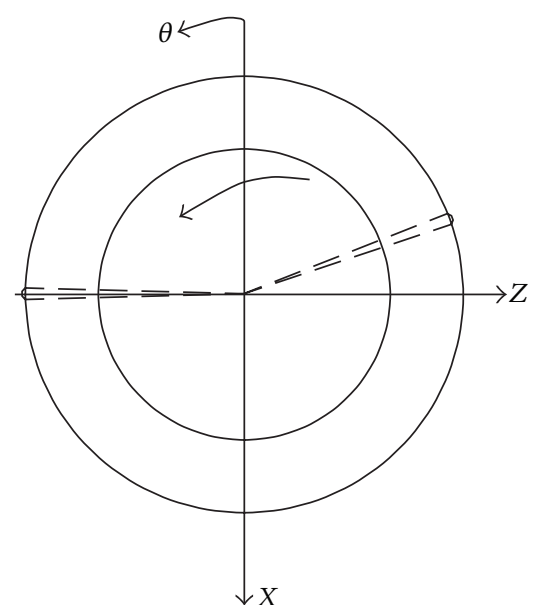

Figure 6: Hz-Up configuration of two-groove oil journal bearing.

varied at $5^{\circ}$ interval (Figure 7). Dn-10-Up-10 configuration means that the left one is $10^{\circ}$ down and the right groove is $10^{\circ}$ above the horizontal as shown in Figure 7.

Group-VII: Dn-Hz configuration: the left groove is $\left(5^{\circ}\right.$ to $80^{\circ}$ ) below the horizontal position, and the other groove is in horizontal position (Figure 8); groove position is varied at $5^{\circ}$ interval (Figure 8). Dn- $10 \mathrm{~Hz}$ configuration means that the left groove is $10^{\circ}$ below the horizontal and the right one is horizontal as shown in Figure 8.

Group-VIII: Hz-Dn configuration: the left groove is in horizontal position, and the other groove is $\left(5^{\circ}\right.$ to $\left.80^{\circ}\right)$ below the horizontal position; groove position is varied at $5^{\circ}$ interval (Figure 9). Hz-Dn-10 configuration means that the left groove is horizontally placed while the right one is $10^{\circ}$ below the horizontal as shown in Figure 9.

Group-IX: Up-Dn configuration: the left groove is $\left(5^{\circ}\right.$ to $\left.80^{\circ}\right)$ above horizontal position, and the other groove is $\left(5^{\circ}\right.$ to $\left.80^{\circ}\right)$ below the horizontal position; groove position is varied at $5^{\circ}$ interval (Figure 10). Up10-Dn-10 configuration means that the left groove is $10^{\circ}$ above the horizontal and the right one is $10^{\circ}$ below the horizontal as shown in Figure 10.

To ascertain the size of the groove for better performance, a comparison of nondimensional load is made for different groove angles as shown in Table 2 . It has been observed that the load carrying capacity is slightly higher with $10^{\circ}$ groove angles in comparison with $20^{\circ}$ and $30^{\circ}$ groove angles (Table 2) in case of two axial groove bearings. Therefore, $10^{\circ}$ groove angles are considered throughout the analysis.

A code has been developed to calculate the steady state and dynamic characteristics for given values of $L / D$ ratios and groove locations (group-I to group-IX), which is subsequently used for obtaining optimum groove locations for different objective functions. An optimum groove location has been obtained depending on maximization of load, flow 


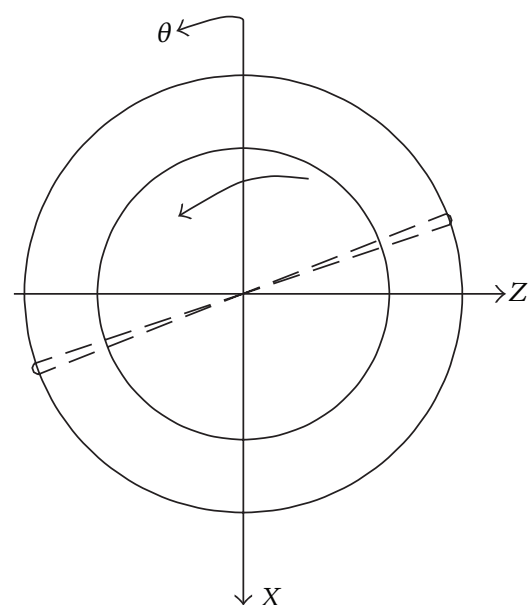

FIGURE 7: Dn-Up configuration of two-groove oil journal bearing.

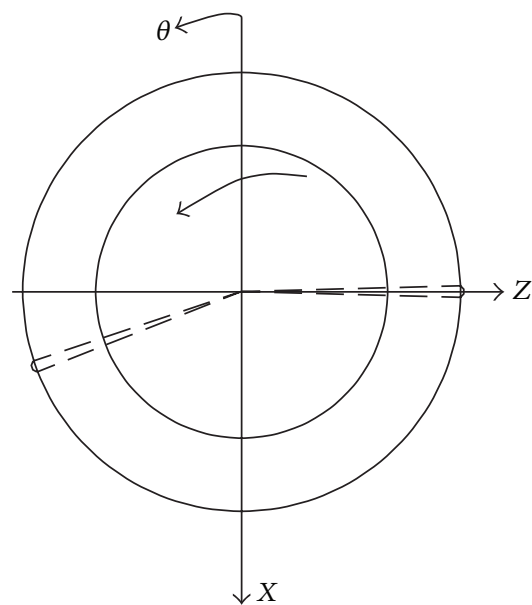

FIGURE 8: Dn-Hz configuration of two-groove oil journal bearing.

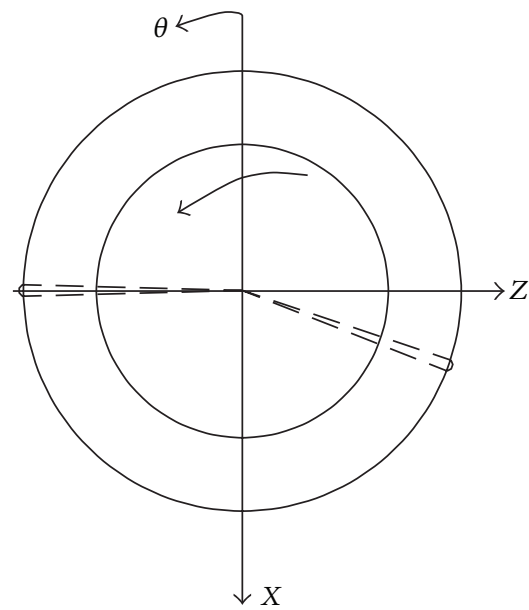

FIgURE 9: Hz-Dn configuration of two-groove oil journal bearing.

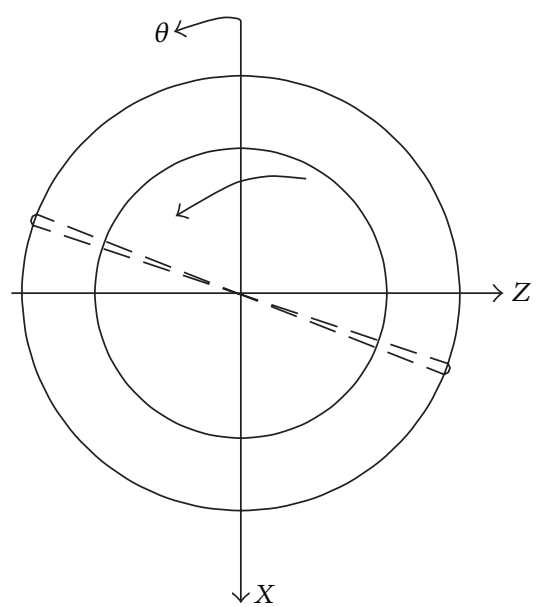

FIgURE 10: Up-Dn configuration of two-groove oil journal bearing.

TABLE 2: Comparison of nondimensional load values using $10^{\circ}, 20^{\circ}$, and $30^{\circ}$ groove angles.

\begin{tabular}{|c|c|c|c|}
\hline \multirow{2}{*}{$\varepsilon$} & \multicolumn{3}{|c|}{$W$} \\
\hline & $10^{\circ}$ groove & $20^{\circ}$ groove & $30^{\circ}$ groove \\
\hline 0.200 & 0.077 & 0.074 & 0.0715 \\
\hline 0.400 & 0.1865 & 0.181 & 0.175 \\
\hline 0.600 & 0.406 & 0.399 & 0.389 \\
\hline 0.800 & 1.135 & 1.123 & 1.107 \\
\hline
\end{tabular}

TABLE 3: Comparison of GA and SQP results.

\begin{tabular}{lcc}
\hline$\varepsilon$ & $\begin{array}{c}\text { Objective function value (minimum friction variable) } \\
\text { GA results }\end{array}$ & SQP results \\
\hline 0.100 & 25.841 & 25.841 \\
0.200 & 12.575 & 12.575 \\
0.300 & 7.991 & 7.991 \\
0.400 & 5.603 & 5.603 \\
0.500 & 4.050 & 4.050 \\
0.600 & 3.023 & 3.023 \\
0.700 & 2.146 & 2.146 \\
0.800 & 1.501 & 1.501 \\
0.900 & 0.358 & 0.358
\end{tabular}

and mass parameter, and minimization of friction with the help of Genetic Algorithm (GA) toolbox of MatLab. The obtained results from (GA) have been compared with the results obtained using sequential quadratic programming (SQP).

The optimum value of fitness function obtained corresponding to minimization of friction variable has been tabulated for both GA and SQP in Table 3.

Similarly maximum load, maximum flow, and maximum mass parameter values are also found to match both methods. It has been observed as stated above that the results using both methods are found to be the same. However, GA has been used in this work as GA, being a heuristic search and optimization technique inspired by natural evolution, has 


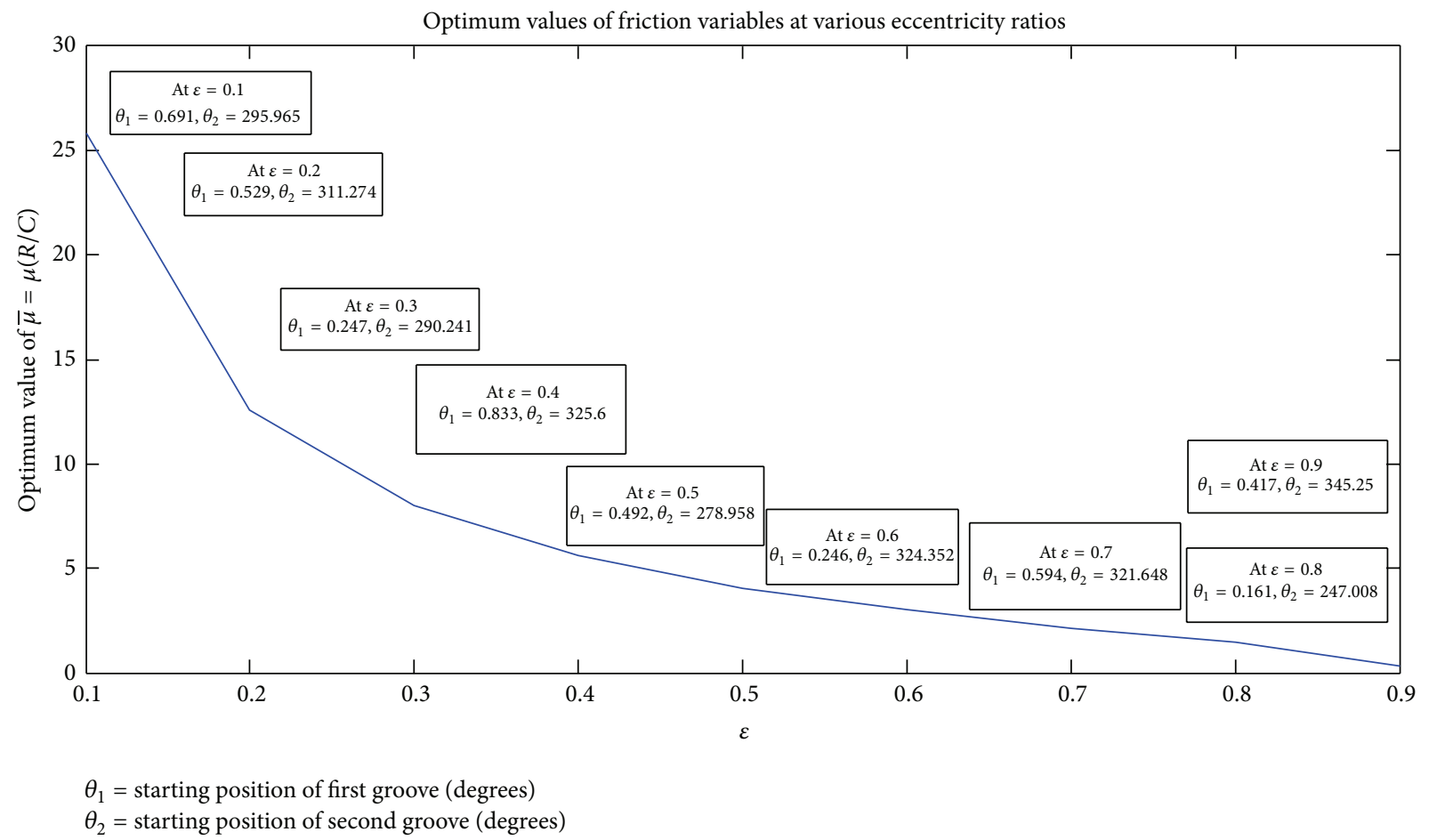

FIGURE 11: Variation of friction variable at optimum grooving location for different $\varepsilon$.

been successfully applied to a wide range of real-world problems of significant complexity $[3,9]$. It has been suggested that heuristic optimization provides a robust and efficient approach for solving complex real-world problems [5].

Initially a single objective function has been taken up. The generic algorithm convergence rate to true optima depends on the probability of crossover and mutation, on one hand, and the maximum generation, on the other hand. In order to preserve a few very good strings and reject low-fitness strings, a high crossover probability is preferred. The mutation operator helps to retain the diversity in the population but disrupts the progress towards a converged population and interferes with beneficial action of the selection and crossover. Therefore, a low probability, $0.001-0.1$, is preferred. The genetic algorithm updates its population on every generation, with a guarantee of better or equivalent fitness strings. For wellbehaved functions, 30-40 generations are sufficient. For steep and irregular functions, 50-100 generations are preferred [2]. Considering these factors, a population size of 50, mutation probability of 0.1 , and a cross over probability of 0.8 have been selected.

The optimum groove locations for minimum nondimensional friction variable, nondimensional load, nondimensional flow, and mass parameter at different $\varepsilon$ are shown in Figures $11,12,13$, and $14 . \theta_{1}$ and $\theta_{2}$ are the starting positions of first and second groove, respectively, in degrees.

From the results shown in Figures 11 through 14, it has been observed that first groove location remains near $0^{\circ}$, whereas the second groove location varies with eccentricity ratios in all the cases. Variations of the second groove location are different for different objective functions.
TABLE 4: The optimum configurations combining all the objective functions at a time.

\begin{tabular}{lcc}
\hline$\varepsilon$ & $\theta_{1}$ & $\theta_{2}$ \\
\hline 0.100 & 0.346 & 336.325 \\
0.200 & 0.141 & 196.512 \\
0.300 & 1.238 & 208.919 \\
0.400 & 0.469 & 240.390 \\
0.500 & 0.281 & 241.176 \\
0.600 & 0.382 & 231.287 \\
0.700 & 0.785 & 222.092 \\
0.800 & 0.476 & 343.712 \\
\hline
\end{tabular}

Similarly by combining all the objective functions at a time the optimum configurations obtained is tabulated (Table 4).

It has been observed from the tabulated results in Table 4 that the staring position of the first groove at different eccentricity ratios for multiobjective function remains near to $0^{\circ}$, whereas second groove location varies for different eccentricity ratios. This indicates that second groove location is more sensitive compared to the first groove location.

If the three variables, namely, eccentricity ratio $(\varepsilon)$, starting angles of the first groove $\left(\theta_{1}\right)$, and the second groove $\left(\theta_{2}\right)$, are taken as chromosome (Table 5), then the optimum results obtained for friction, flow, load, and mass parameter are shown in Figures 15, 16, 17, and 18. The figures include plots of best fitness as well as mean fitness. Genetic algorithm works on a population of individuals. So, mean is the mean fitness for the entire population at a particular iteration. 


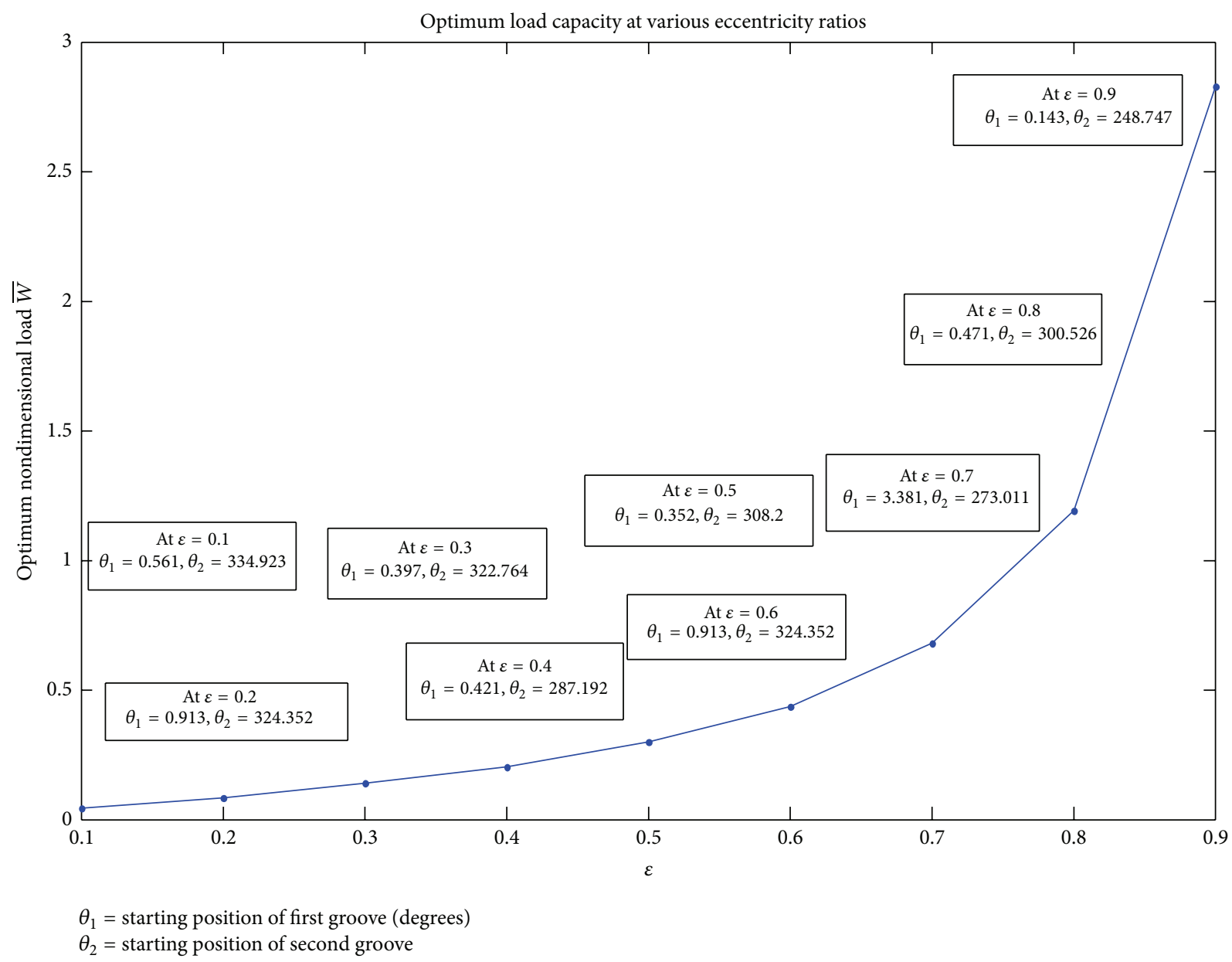

FIGURE 12: Variation of optimum nondimensional load at different eccentricity ratios.

TABLE 5: Variable bounds for the bearing problem.

\begin{tabular}{lcc}
\hline Variable & Lower bound & Upper bound \\
\hline$\varepsilon$ & 0.100 & 0.900 \\
Starting angle of first groove $\left(\theta_{1}\right)$ & $0^{\circ}$ & $180^{\circ}$ \\
Starting angle of second groove $\left(\theta_{2}\right)$ & $170^{\circ}$ & $350^{\circ}$ \\
\hline
\end{tabular}

TABLE 6: Optimum location considering different objectives.

\begin{tabular}{lccc}
\hline Optimum location for objectives & $\varepsilon$ & $\theta_{1}$ & $\theta_{2}$ \\
\hline Minimum friction variable & 0.900 & 0 & 232.906 \\
Maximum flow & 0.899 & 5.660 & 301.960 \\
Maximum load carrying capacity & 0.899 & 0.626 & 308.230 \\
Maximum mass parameter & 0.811 & 0.890 & 308.230 \\
$\begin{array}{l}\text { Optimization of the combined } \\
\text { objectives }\end{array}$ & 0.268 & 3.670 & 349.990
\end{tabular}

Again by combining all the objective functions at a time the fitness value plot has been obtained as shown in Figure 19. Here weighted sum method has been used to combine all the objectives. There are three objectives to be maximized when one has to be minimized. The objectives to be maximized are made negative, and then the weighted sum of all the four objective functions has been taken making the multiobjective problem of minimization type. Since there are four parameters weights equal to 0.25 is used.

The optimum locations for each objective function including that of multi-objective function have been shown in Table 6. From the above analysis, it has been observed that groove locations for various objective functions are different. The first groove varies between $0^{\circ}$ to $5.66^{\circ}$, and the second groove locations for maximum load carrying capacity and maximum mass parameter are the same. Second groove location for minimum friction variable is the least and for multiobjective function is the highest. Another interesting observation is that when the corresponding eccentricity ratios for individual objective functions are high enough, it is much less for multiobjective function.

After carefully looking at the results presented above, it appears that one may get near optimal results by placing a single groove and eliminating the second groove entirely. In view of this, an attempt has been made to find the optimum groove location for a single-grove bearing and compared with two-groove cases for each of the objective functions as presented in Table 7 . Since the results are found to be quite interesting, therefore, it would be pertinent to go through 


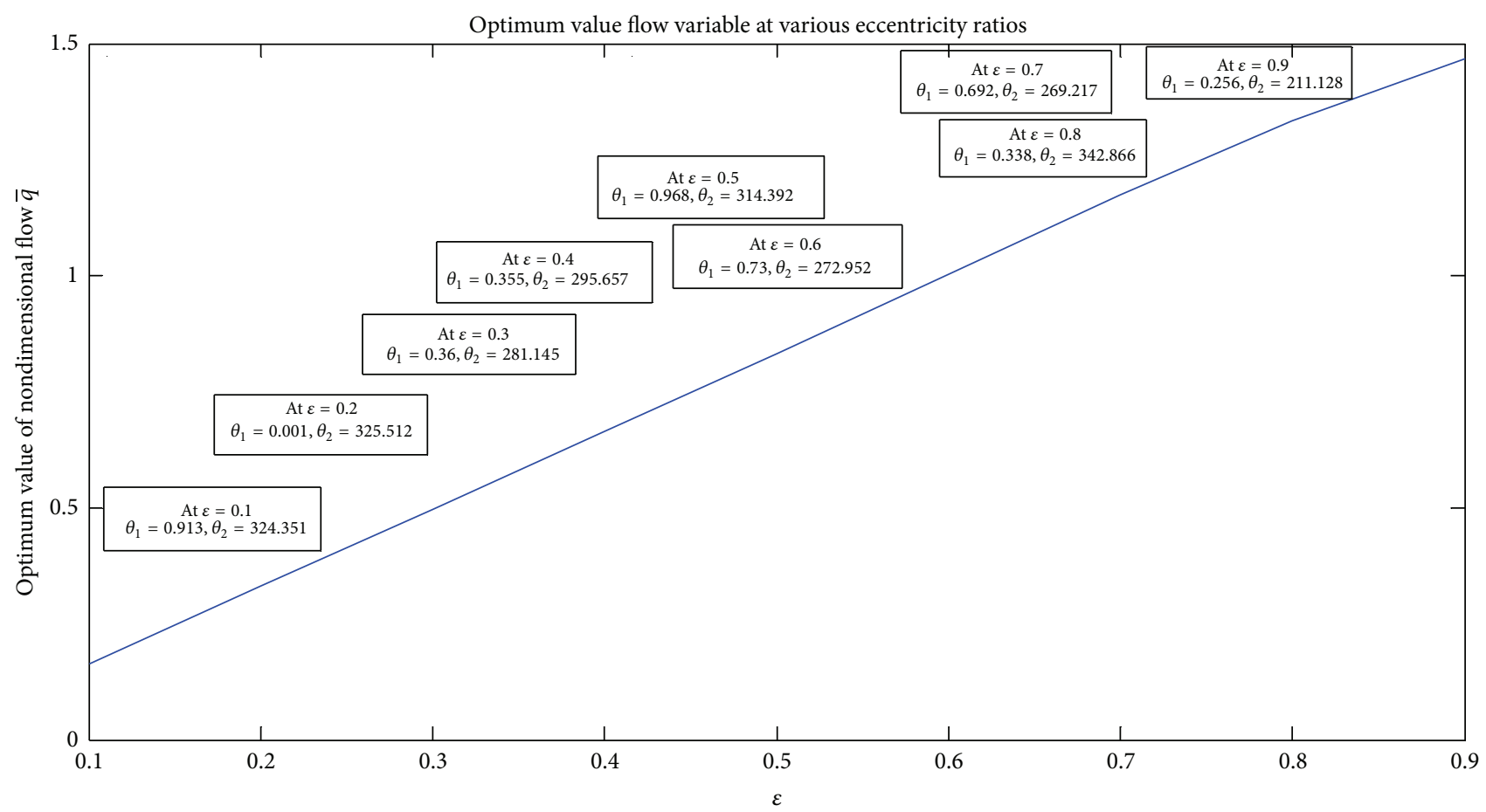

$\theta_{1}=$ starting position of first groove (degrees)

$\theta_{2}=$ starting position of second groove (degrees)

FIGURE 13: Variation of flow at optimum grooving location for different eccentricity ratios.

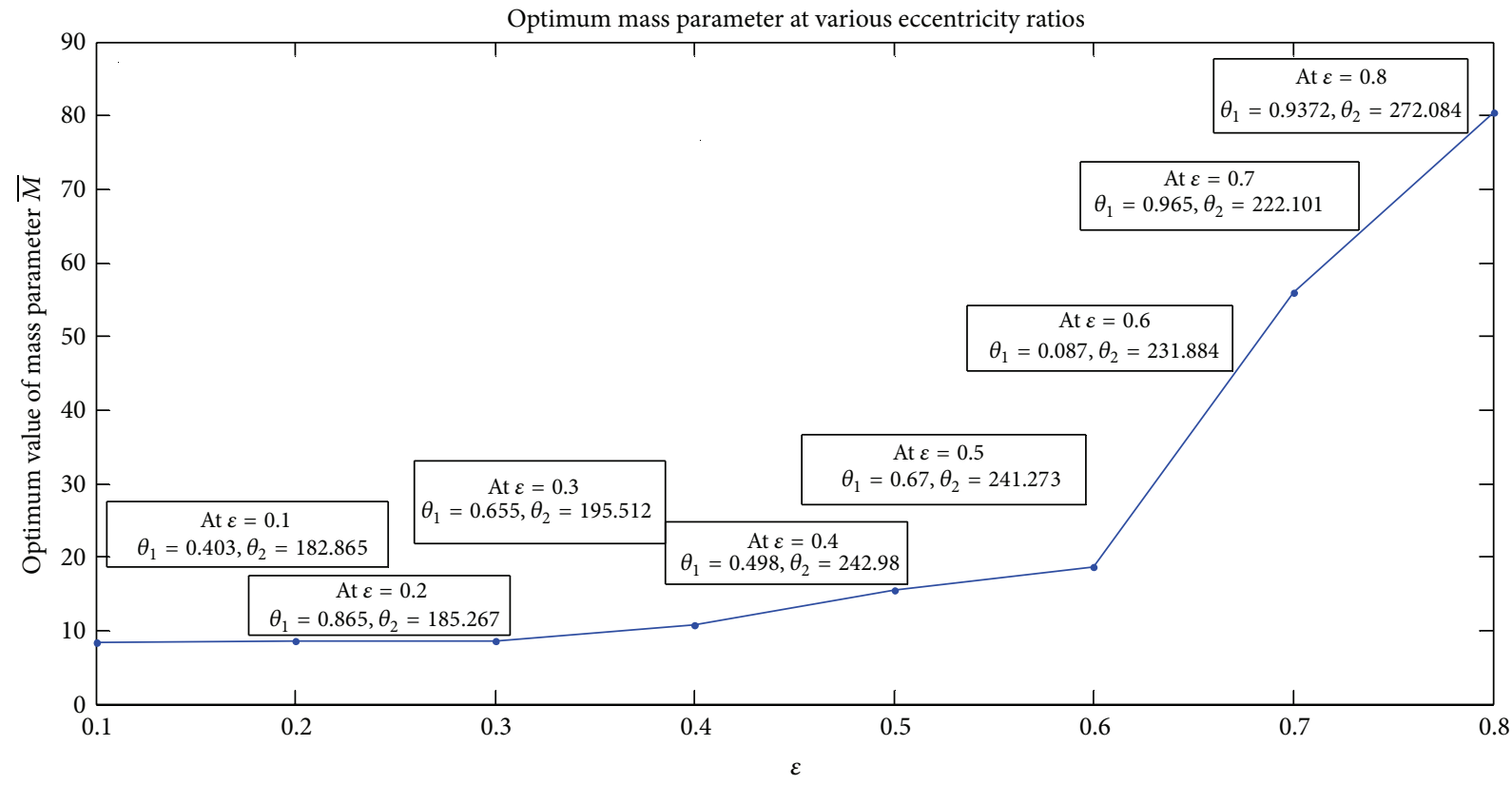

$\theta_{1}=$ starting position of first groove (degrees)

$\theta_{2}=$ starting position of second groove (degrees)

FIGURE 14: Variation of mass parameter at optimum grooving location for different eccentricity ratios. 


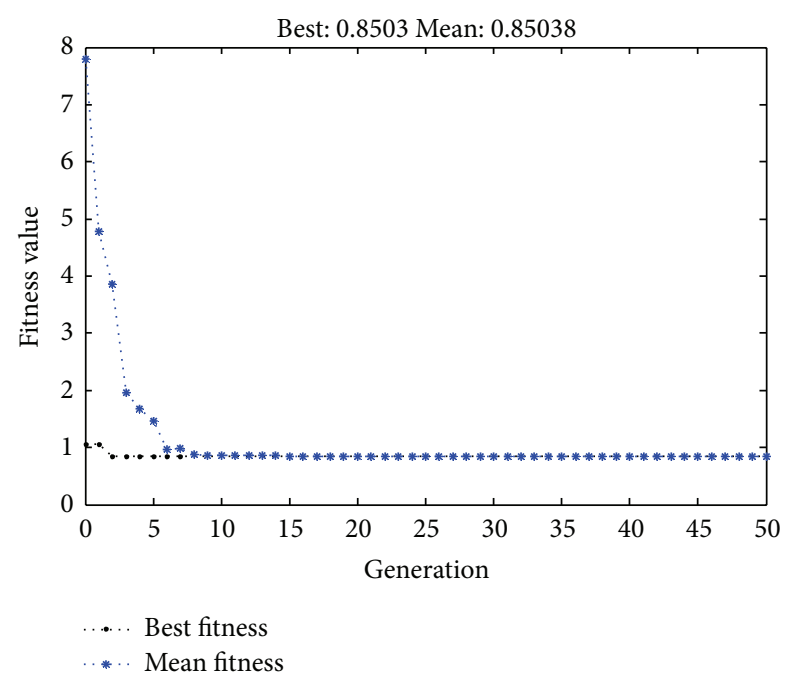

FIGURE 15: Fitness value considering friction variable as objective function.

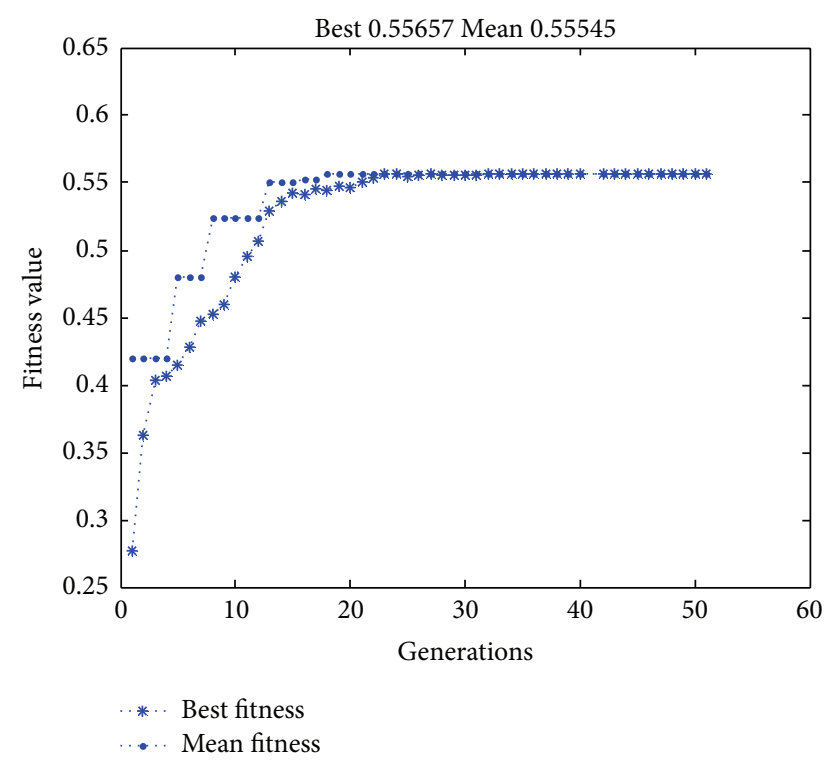

FIGURE 16: Fitness value considering flow as objective function.

relevant literature first and then taking up the study to find whether single groove or two grooves would enhance the bearing performance. The authors would like to keep this for future study in detail.

A dimensional example has been shown below to demonstrate how to convert the nondimensional parameters to dimensional parameters.

Let $D=100 \mathrm{~mm}, N=3000 \mathrm{rpm}, W=15 \mathrm{kN}, L / D=$ $1.0, C / R=0.001$.

So, $C=50 \times 10^{-6} \mathrm{~m}$.

Taking minimum film thickness as $h_{o}=25 \times 10^{-6} \mathrm{~m}$, one gets $h_{o} / C=0.5$.

Hence, $\varepsilon=0.5$.

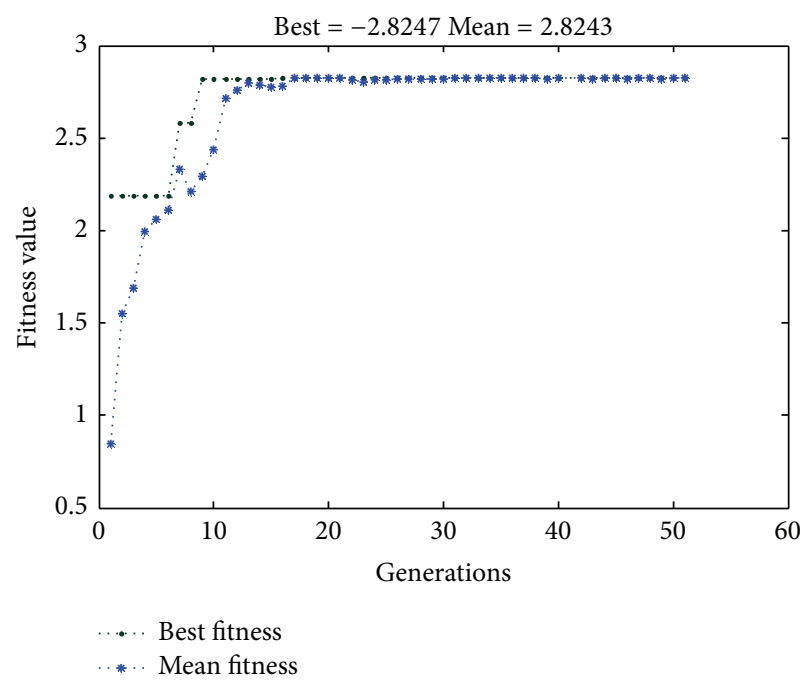

FIGURE 17: Fitness value considering load as objective function.

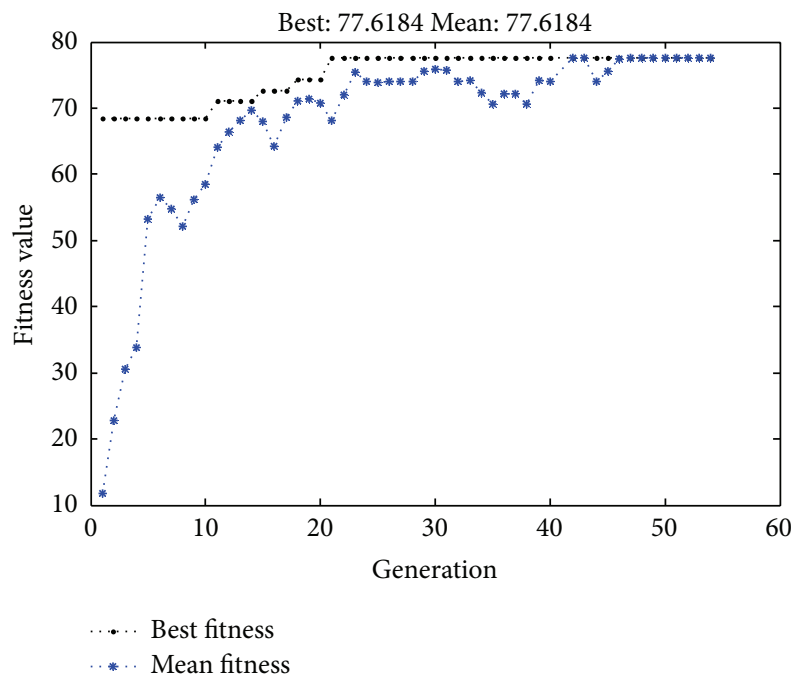

FIGURE 18: Fitness value considering mass parameter as objective function.

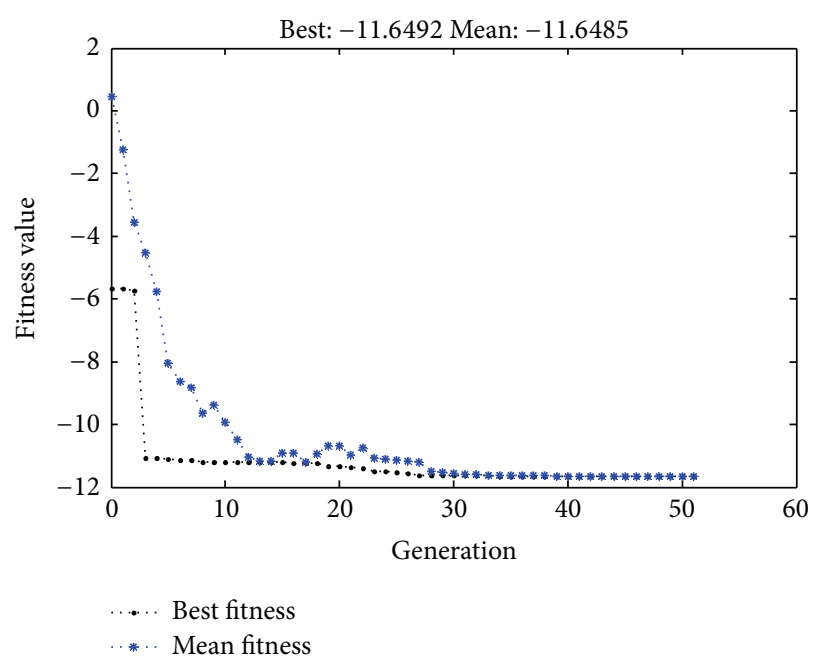

FIGURE 19: Fitness value combining all the objective function. 
TABLE 7: Comparison of optimum locations of grooves for two-groove and single-groove bearings.

\begin{tabular}{|c|c|c|c|c|}
\hline Comparison & Objective function & $\varepsilon$ & $\theta_{1}$ & $\theta_{2}$ \\
\hline For two groove & \multirow{2}{*}{ Minimum friction variable } & 0.900 & 0 & 232.906 \\
\hline For single groove & & 0.100 & 180 & - \\
\hline For two groove & \multirow{2}{*}{ Maximum flow } & 0.899 & 5.66 & 301.96 \\
\hline For single groove & & 0.100 & 180 & - \\
\hline For two groove & \multirow{2}{*}{ Maximum load } & 0.899 & 0.626 & 308.23 \\
\hline For single groove & & 0.657 & 349.038 & - \\
\hline For two groove & \multirow{2}{*}{ Maximum mass parameter } & 0.811 & 0.890 & 308.23 \\
\hline For single groove & & 0.704 & 223.435 & - \\
\hline For two groove & \multirow{2}{*}{ Optimization of all the combined objectives } & 0.268 & 3.67 & 349.99 \\
\hline For single groove & & 0.657 & 349.038 & - \\
\hline
\end{tabular}

TABLE 8: Conversion of nondimensional results to dimensional ones.

\begin{tabular}{lcccc}
\hline Eccentricity ratio & Objective function & $\begin{array}{c}\text { Optimum groove } \\
\text { locations }\end{array}$ & Present nondimensional result & Dimensional values \\
\hline \multirow{4}{*}{ Maximization of flow } & $\begin{array}{c}\theta_{1}=0.968 \\
\theta_{2}=314.392\end{array}$ & 0.8329 (optimum flow variable) & $\begin{array}{c}\text { Flow, } \\
\theta_{1}=0.492\end{array}$ \\
& Minimization of friction & 4.050 (optimum friction variable) & $\begin{array}{c}\text { Coefficient of Friction, } \\
\theta_{2}=278.95\end{array}$ \\
& & & $\mu=4.050 \times 10^{-3}$ \\
\hline
\end{tabular}

For $\varepsilon=0.5$, optimum friction variable and optimum flow variables are shown in Table 8 along with optimum groove locations. These non-dimensional results are converted to dimensional parameters, namely, flow in $\mathrm{m}^{3} / \mathrm{s}$ and coefficient of friction by using the above data. These values may further be used to estimate the friction force, temperature rise, and so forth.

\section{Conclusion}

From the results presented here, it can be inferred that the second groove location is sensitive to the type of objective function whereas the first groove is more or less the same for any objective function. The practice and the notion of convenience of keeping groove positions $180^{\circ}$ apart need to be thoroughly looked into as the present results show that optimum groove locations are not $180^{\circ}$ apart for any of the objective functions considered in the present work. Experimental verification of the present result may lead to a new approach of production of bearings with optimum groove locations; however, it is beyond the scope of the present work and hopefully experimentalists have a problem in hand.

\section{Appendix}

For the purpose of validation of results the steady state characteristics of two-groove oil journal bearing having $20^{\circ}$ groove angles placed in horizontal position for $L / D=1$ are compared with the published results [2] as shown in Table 9. The present results are found to be fairly in good agreement with [2].
TABLE 9: Comparison of present results with [2] for $L / D=1$ and $20^{\circ}$ axial groove for groove in the horizontal position.

\begin{tabular}{lcc}
\hline$\varepsilon$ & $S$ & $\phi$ \\
& Present [Ref] & Present [Ref] \\
\hline 0.103 & $1.453[1.470]$ & $75.860[75.990]$ \\
0.150 & $0.980[0.991]$ & $70.462[71.580]$ \\
0.224 & $0.629[0.635]$ & $63.4598[63.540]$ \\
0.352 & $0.352[0.358]$ & $56.100[55.410]$ \\
0.460 & $0.232[0.235]$ & $49.925[49.270]$ \\
0.559 & $0.157[0.159]$ & $45.1075[44.330]$ \\
0.650 & $0.106[0.108]$ & $40.120[39.720]$ \\
0.734 & $0.070[0.071]$ & $35.432[35.160]$ \\
0.773 & $0.0562[0.056]$ & $33.160[32.860]$ \\
0.811 & $0.043[0.044]$ & $30.614[-]$ \\
0.883 & $0.023[0.024]$ & $25.142[25.020]$ \\
\hline
\end{tabular}

\section{Notations}

C: $\quad$ Radial clearance $(\mathrm{m})$

$D$ : Diameter of the journal (m)

$L: \quad$ Length of the bearing $(\mathrm{m})$

$R: \quad$ Bearing radius $(\mathrm{m})$

$e: \quad$ Eccentricity $(\mathrm{m})$

$\varepsilon: \quad$ Eccentricity ratio $=e / C$

$\eta$ : Coefficient of absolute viscosity of the lubricant (Pa-s)

$\mu, \bar{\mu}$ : Coefficient of friction, friction variable $=\mu(R / C)$

$N$ : Speed of the journal in r.p.s

$\phi$ : Bearing attitude angle

$h$ : Film thickness $(\mathrm{m})$ $=C(1+\varepsilon \cos \theta)$ 


\begin{tabular}{|c|c|}
\hline$h:$ & $\begin{array}{l}\text { Nondimensional film thickness } \\
=h / C\end{array}$ \\
\hline$\theta_{1}:$ & Position of starting of the groove \\
\hline$\theta_{2}:$ & Position of end of the groove \\
\hline$U:$ & Sliding speed \\
\hline$p:$ & Steady state pressure $(\mathrm{Pa})$ \\
\hline $\bar{p}:$ & $\begin{array}{l}\text { Nondimensional steady state } \\
\text { pressure }=p C^{2} / 6 \eta U R\end{array}$ \\
\hline$W:$ & Load carrying capacity (N) \\
\hline $\bar{W}:$ & $\begin{array}{l}\text { Nondimensional load carrying } \\
\text { capacity }=W C^{2} / 6 \eta U R^{2} L\end{array}$ \\
\hline$X:$ & Vertical direction \\
\hline$Z:$ & Horizontal direction \\
\hline$W_{X}:$ & $\begin{array}{l}\text { Vertical component (in } X \\
\text { direction) of the resultant load }\end{array}$ \\
\hline$W_{Z}:$ & $\begin{array}{l}\text { Vertical component (in } Z \\
\text { direction) of the resultant load }\end{array}$ \\
\hline$P:$ & $\begin{array}{l}\text { Load per unit bearing area } \\
=W / L D\end{array}$ \\
\hline$S:$ & $\begin{array}{l}\text { Sommerfeld number } \\
=(\eta N / P)(R / C)^{2}\end{array}$ \\
\hline $\bar{q}_{z}:$ & $\begin{array}{l}\text { Nondimensional flow coefficient, } \\
\left(\bar{q}_{z}=2 Q / U L C\right)\end{array}$ \\
\hline $\bar{p}_{1}, \bar{p}_{2}:$ & Perturbed pressures \\
\hline & $\begin{array}{l}\text { Perturbed eccentricity ratio and } \\
\text { attitude angle around the steady } \\
\text { state value } \varepsilon_{0}, \phi_{0}\end{array}$ \\
\hline$K_{X X}, K_{Z Z}, K_{X Z}, K_{Z X}:$ & Stiffness coefficients $(\mathrm{N} / \mathrm{m})$ \\
\hline $\bar{K}_{X X}, \bar{K}_{Z Z}, \bar{K}_{X Z}, \bar{K}_{Z X}:$ & $\begin{array}{l}\text { Nondimensional stiffness } \\
\text { coefficients }=K_{i j} C / W \text {, where } \\
i=X, Z \text { and } j=X, Z\end{array}$ \\
\hline$\underline{D}_{X X}, D_{Z Z}, D_{X Z}, D_{Z X}:$ & Damping coefficient $(\mathrm{N} \cdot \mathrm{s} / \mathrm{m})$ \\
\hline $\bar{D}_{X X}, \bar{D}_{Z Z}, \bar{D}_{X Z}, \bar{D}_{Z X}:$ & $\begin{array}{l}\text { Nondimensionaldamping co } \\
\text { efficient }=C_{i j} C \omega / W \text {, where } \\
i=X, Z \text { and } j=X, Z\end{array}$ \\
\hline$t:$ & Time (s) \\
\hline$\omega, \omega_{p}:$ & $\begin{array}{l}\text { Journal rotational speed }(\mathrm{rad} / \mathrm{s}) \text {, } \\
\text { frequency of journal vibration }\end{array}$ \\
\hline$\tau:$ & Nondimensional time, $\tau=\omega_{p} t$ \\
\hline$\lambda:$ & Whirl ratio $=\omega_{p} / \omega$ \\
\hline$M, \bar{M}:$ & $\begin{array}{l}\text { Rotor mass }(\mathrm{kg}) \text {, mass parameter, } \\
\bar{M}=M C \omega^{2} / W\end{array}$ \\
\hline()$_{0}:$ & Steady state value. \\
\hline
\end{tabular}

\section{References}

[1] ESDU Items 84031 (and 85028), Calculation Methods for Steadily Loaded Axial Groove Hydrodynamic Journal Bearings, with Superlaminar operation, December 1985, 1984.

[2] P. Klit and J. W. Lund, "Calculation of the dynamic coefficients of a journal bearing, using a variational approach," Journal of Tribology, vol. 108, no. 3, pp. 421-425, 1986.

[3] D. T. Gethin and M. K. I. El Deihi, "Effect of loading direction on the performance of a twin-axial groove cylindrical-bore bearing," Tribology International, vol. 20, no. 4, pp. 179-185, 1987.

[4] W. B. Rowe and D. Koshal, "A new basis for the optimization of hybrid journal bearings," Wear, vol. 64, no. 1, pp. 115-131, 1980.
[5] Y. J. Lin and S. T. Noah, "Using genetic algorithms for the optimal design of fluid journal bearing," in Proceedings of the ASME Design Engineering Technical Conferences, pp. 12-15, 1999.

[6] H. Hashimoto and K. Matsumoto, "Improvement of operating characteristics of high-speed hydrodynamic journal bearings by optimum design: part I-formulation of methodology and its application to elliptical bearing design," Journal of Tribology, vol. 123 , no. 2, pp. 305-312, 2001.

[7] S. Boedo and S. L. Eshkabilov, "Optimal shape design of steadily loaded journal bearings using genetic algorithms," Tribology Transactions, vol. 46, no. 1, pp. 134-143, 2003.

[8] H. Hirani, "Multiobjective optimization of a journal bearing using the Pareto optimality concept," Proceedings of the Institution of Mechanical Engineers J, vol. 218, no. 4, pp. 323-336, 2004.

[9] B. David, D. R. Bull, and M. Ralph, "An overview of genetic algorithms: part 2, research topics," University Computing, vol. 25, no. 4, pp. 170-181, 1993.

[10] J. McCall, "Genetic algorithms for modelling and optimisation," Journal of Computational and Applied Mathematics, vol. 184, no. 1, pp. 205-222, 2005.

[11] B. J. Hamrock, Fundamentals of Film Lubrication, Mc GrawHill, NewYork, NY, USA, 1994. 

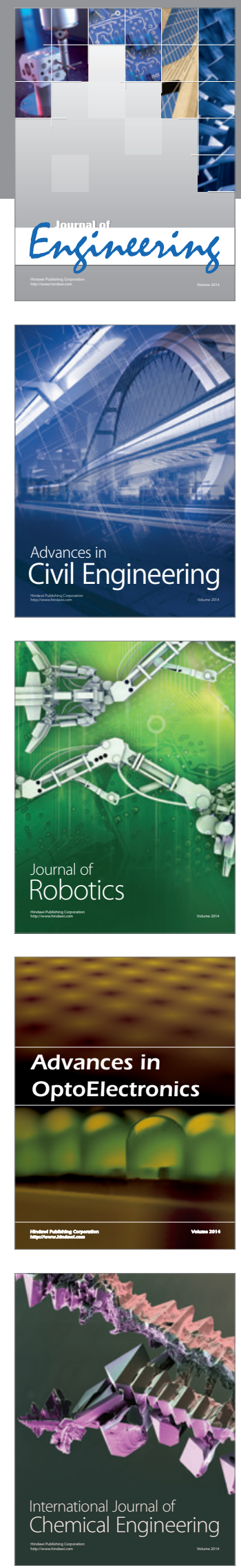

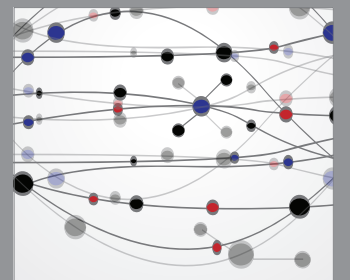

The Scientific World Journal
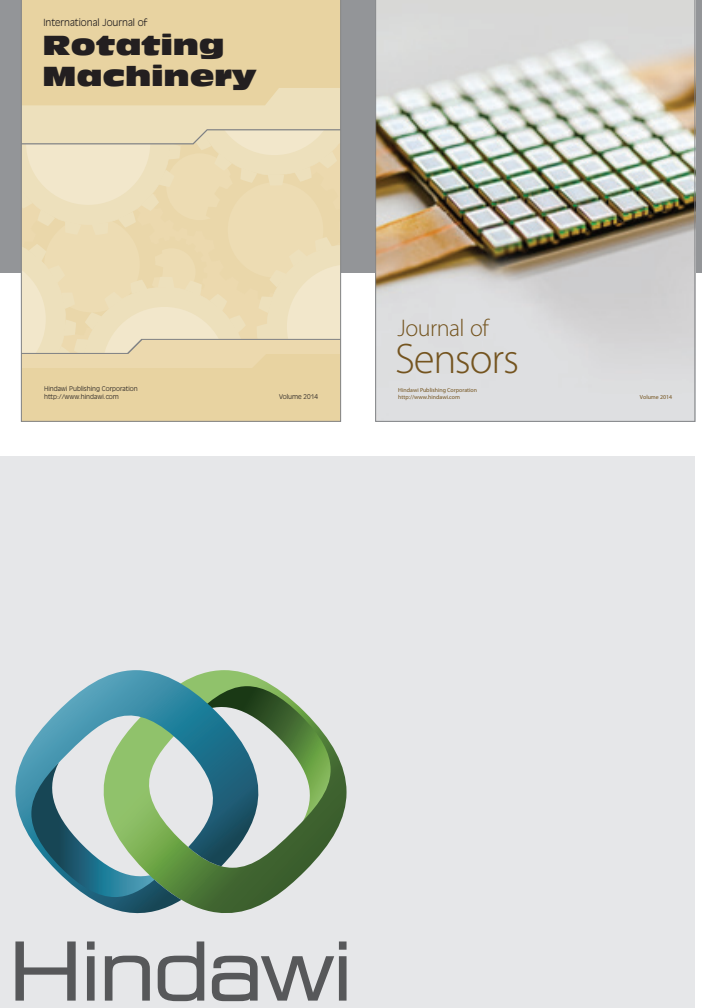

Submit your manuscripts at http://www.hindawi.com
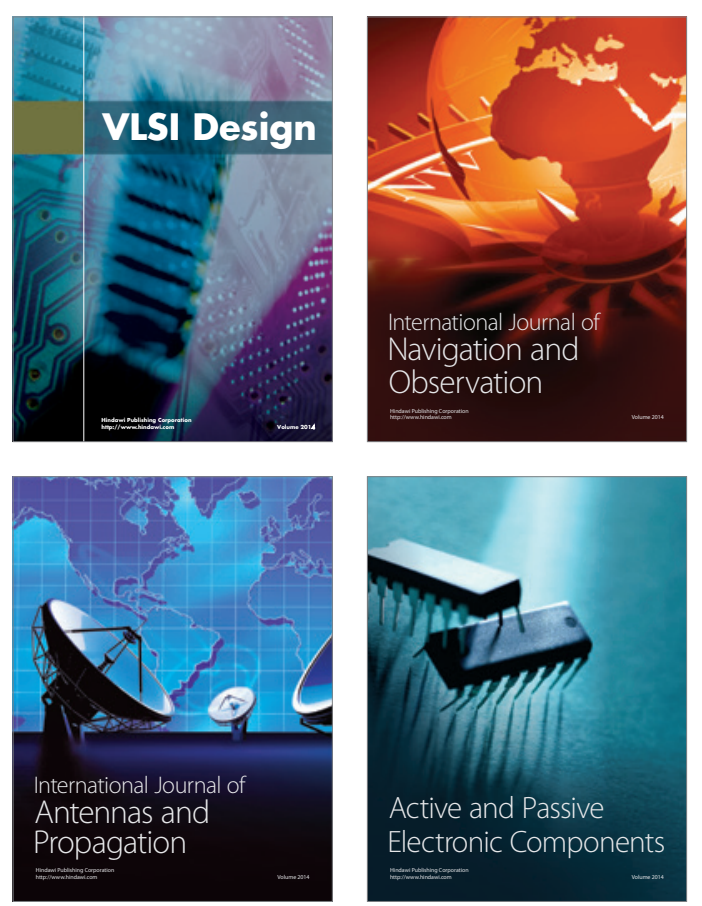
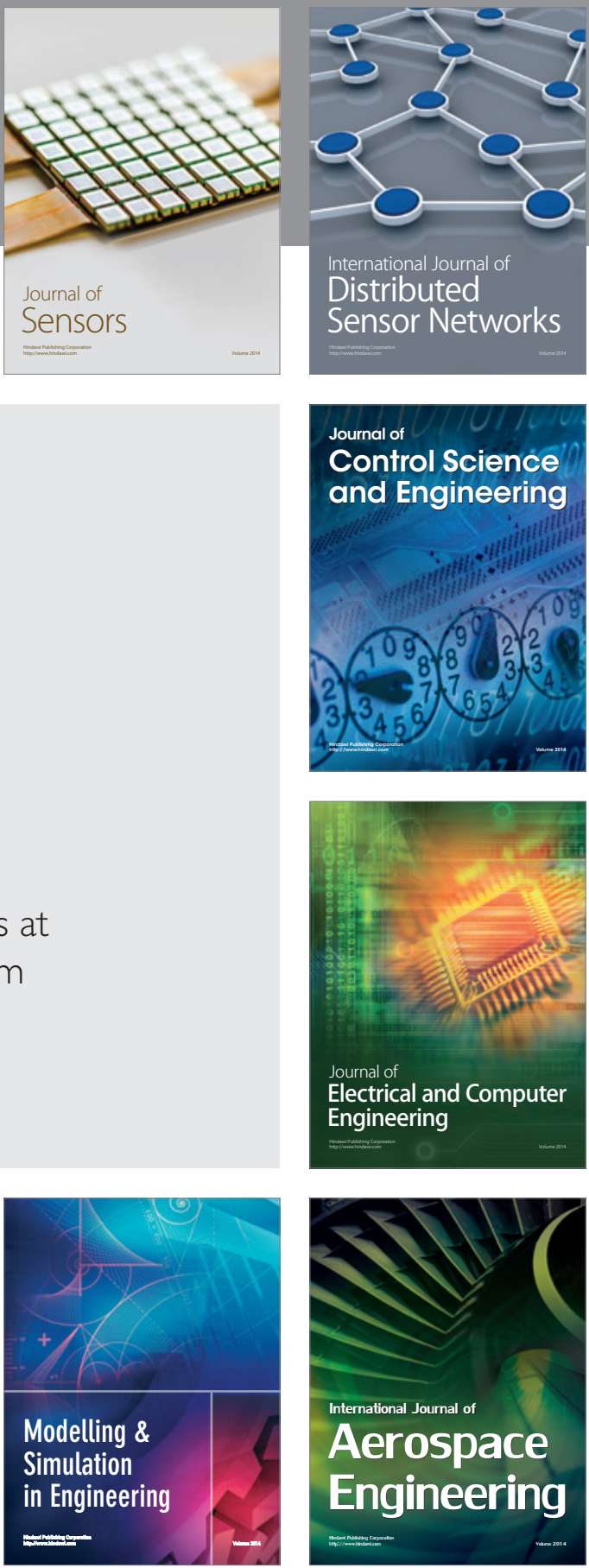

Journal of

Control Science

and Engineering
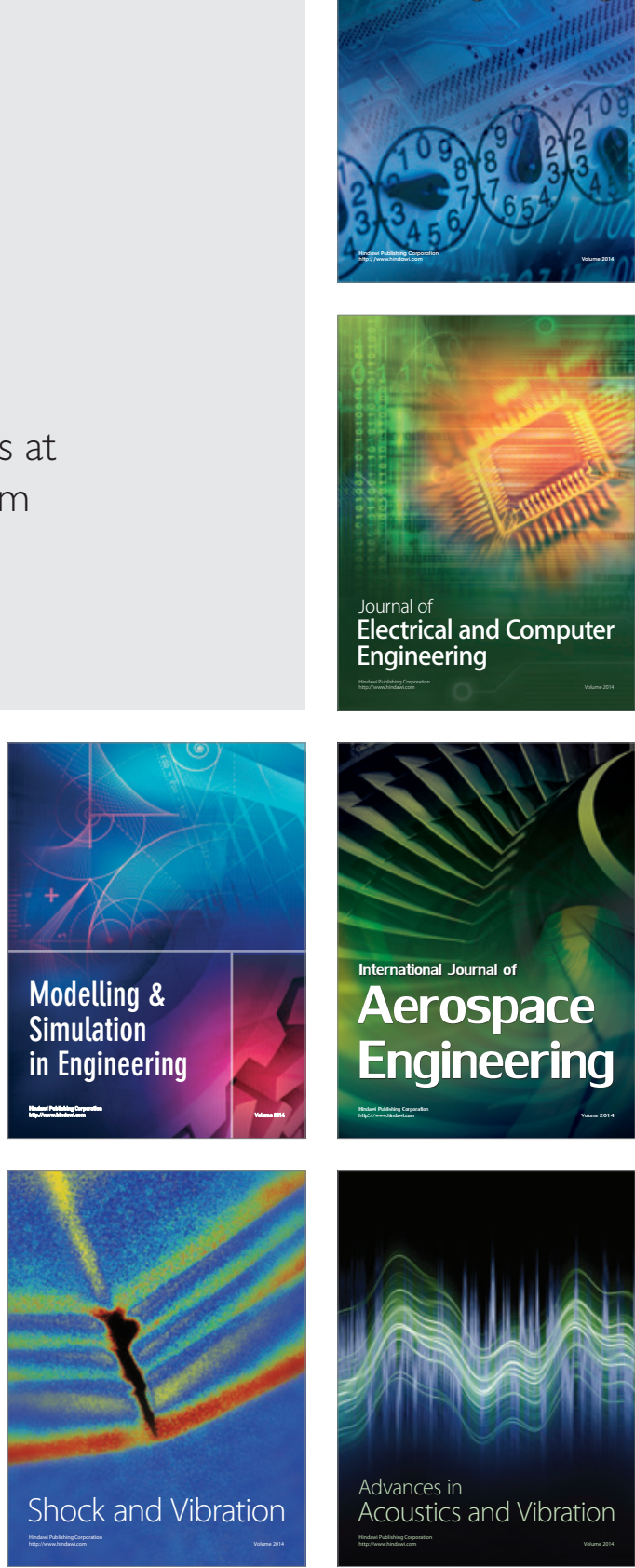